\title{
Nosocomial Infections at Three Regional Tertiary Hospitals in Trinidad and Tobago
}

\section{Camille Elliott $^{1^{*}}$, A Justiz-Vaillant ${ }^{2}$}

${ }^{* 1}$ Department of Para- Clinical Science. Unit of Pathology and Microbiology. Faculty of Medical Science. Mount Hope Hospital. The University of the West Indies, St. Augustine.

${ }^{2}$ Department of Para- Clinical Sciences. (Pathology and Microbiology Unit). University of the West Indies.Eric Williams Medical Complex, Mt. Hope Hospital, Champs Fleurs, Trinidad and Tobago, West Indies.

*Corresponding Author: Camille Elliott, Department of Para- Clinical Sciences. (Pathology and Microbiology Unit). University of the West Indies. Eric Williams Medical Complex, Mt. Hope Hospital, Champs Fleurs, Trinidad and Tobago, West Indies. Cell: 1(868) $377-3804$.

Received date: February 07, 2020; Accepted date: March 16, 2020; published date: March 20, 2020

Citation: Camille Elliott, A Justiz-Vaillant. (2020) Nosocomial Infections at Three Regional Tertiary Hospitals in Trinidad and Tobago.

Biomedical Research and Clinical Reviews. 1(1); DOI: 10.31579/2692-9406/001

Copyright:@2020 Camille Elliott, This is an open-access article distributed under the terms of the Creative Commons Attribution License, which permits unrestricted use, distribution, and reproduction in any medium, provided the original author and source are credited.

\section{Abstract}

Objective: The main objectives of this research were to conduct and provide accurate and original findings related to the epidemiological study of nosocomial infections at three regional tertiary hospitals in Trinidad and Tobago. Specifically, the researcher determined; the frequency of nosocomial infections (NI), the frequency of multiple drug resistance among bacterial organisms associated with NI, infection control measures practiced at the research hospitals and the cost of such NI in terms of morbidity and mortality.

Methods: A one-year prospective cross-sectional study was carried out. The nosocomial pathogens were retrieved from the microbiology laboratory. Antimicrobial susceptibility test by the disk diffusion method were done on all bacterial isolates. Data was analysed using SPSS version 20.0.

Results: This research revealed that 450 inpatients suffered nosocomial infections, with thirty (30) mortalities during the twelve (12) months that the study lasted (June 2013 to May 2014) at three regional hospitals of Trinidad and Tobago. The incidence of nosocomial infections was $5.8 \%$ and the nosocomial infection rate was 3.6 per $1000(450 / 126,668)$. The highest rate $(30.1 \%)$ was observed in the Intensive Care Unit (82/272 admissions). The most frequent type of nosocomial infection was Skin and Soft

Tissue Infections 168 (37.3\%). Staphylococcus sp. (22.5\%), Pseudomonas aeruginosa sp. (12.7\%), Acinetobacter (11.8\%) and Klebsiella sp. $(11.6 \%)$ were the most frequently occurring nosocomial pathogens.

Conclusion: Consistency in performing good hygiene practices is vital for reducing the high nosocomial rate found at the research sites. Prediction of these infections is very important as a part of clinical surveillance programs to take preventive measures in advance. The antimicrobial susceptibility pattern rate (ASPR) showed that only $8.3 \%(5 / 60)$ of the isolates were antibiotic-susceptible strains.

Keywords: Antibiotic resistance; cross sectional study; incidence; mortality; Nosocomial Infections (NI) and susceptibility pattern. Short title: Nosocomial Infections at Three Major Hospitals in Trinidad and Tobago, West Indies

Synopsis: This study estimated the rate of nosocomial infections (NI) among patients at three major regional hospitals in Trinidad and Tobago and evaluated the frequency of pathogens associated with nosocomial infections. Approximately 450 of 126, 668 patients had nosocomial infections and the most frequent type of nosocomial pathogens were: Staphylococcus sp. (22.5\%), Pseudomonas aeruginosa sp. (12.7\%), Acinetobacter (11.8\%) and Klebsiella sp. (11.6\%).

\section{Introduction}

Limited studies have been done on nosocomial infections in Trinidad and Tobago. Nosocomial infection is important to study so as to reduce the incidence, mortality and morbidity associated with nosocomial infections. Neonatal and elderly admitted patients are mostly at risk due to their weak immune. Orrett, 2002 indicated in his research in Trinidad and Tobago that 139 NI were identified from 629 admissions to ICU. The main NI was from respiratory tract, $41(29.5 \%)$ followed by surgical wounds, $35(25.2 \%)$, urinary tract, $28(20.1 \%)$ then bloodstream, 24
(17.3\%). From the 165 bacterial organisms, $80 \%$ of these organisms were gram negative bacilli, with Pseudomonas aeruginosa, 48 (36.6\%), being the most common isolate followed by Klebsiella pneumoniae, 27 (20.6\%) then Enterobacter sp. 22 (16.8\%). The major gram positive isolates were Staphylococcus aureus, $23(41.8 \%)$, coagulase-negative Staphylococci, 17 (30.9\%) and Enterococci, 11 (20.0\%) [9]. In Europe, incidences varied from $1 \%$ for all types of nosocomial infections and up to $23.6 \%$ in paediatric ICUs [16]. In the United States of America (USA), the Centre for Disease Control and Prevention (CDC) calculated approximately 1.7 million nosocomial infections from all types of microorganisms resulting in 99,000 deaths annually [17]. The American Thoracic Society/Infectious Disease Society of America (ATS/IDSA) introduced the concept of healthcare-associated pneumonia (HCAP) in 2005 and among its guidelines includes the recommended broadspectrum antibiotics therapy as the treatment of hospital-acquired pneumonia [18]. These nosocomial pathogens affect other organs and tissues including the urinary tract $[3,5,10,19]$, bloodstream [20], brain $[15,21]$, and digestive tract [22] and the skin and soft tissues [23]. 
Prevalence of other multidrug-resistant bacteria are also rising, example vancomycin-resistant Enterococci (VRE) [1, 24] and Clostridium difficile that is an etiological agent of hospital acquired antibioticassociated diarrhoea, causing an estimated 453,000 cases with 29,000 deaths yearly in the USA $[11,25]$.

According to World Health Organization (WHO), health care associated infection (HCAI) is defined as "an infection acquired in hospital by a patient, who was admitted for a reason other than that infection [29]. An infection occurring in a patient in a hospital or other health care facility in whom the infection was not present or incubating at the time of admission. This includes infections acquired in the hospital, but appearing after discharge, and occupational infections among staff of the facility" [30].

The standard criteria that were used along with WHO's definition to confirm nosocomial cases were: the patient had no growth of organism from laboratory culture present at day one of their admission and no signs and symptoms present the same day including temperature spikes, worsening coughing or dyspnea, tachypnea, bronchial rates, breath sounds, vomiting, leukopenia $<4000$, low white blood cell (WBC) counts or leukocytosis, sloppiness at wound, redness, swelling, warmth, hotness around wound area, septicemia, hypotension, dysuria, urgency and tenderness. [31].

The aims of this study were to carry out a hospital microbiological survey for detecting the most prevalent pathogens that were causing nosocomial infections and to study their antimicrobial susceptibility patterns to prevent and control existing outbreaks and to insight in control measures to prevent nosocomial infection in Trinidad and Tobago. The research was a Cross Sectional Study conducted at three major hospitals in Trinidad and Tobago namely; Eric Williams Medical Science Complex, San Fernando General Hospital and Port of Spain General Hospital.

\section{Methods}

\section{Target Population:}

The study population was all patients hospitalized on the following wards: Medical, Surgical, Paediatrics, Intensive Care Unit (ICU) and Obstetrics \& Gynaecology in three public hospitals in Trinidad during the period June 1, 2013 to May 31, 2014.

\section{Inclusion and Exclusion Criteria:}

All patients with features suggestive of nosocomial infections, who were willing to participate and gave their written or verbal consents on ward, were included in the study. Out-patients from accident \& emergency wards and nephrology wards were excluded from this study, if suspected infections were considered community-acquired infections.

\section{Study Design}

This research was a cross-sectional study conducted at three major hospitals in Trinidad and Tobago.

These medical facilities were: Eric Williams Medical Sciences Complex (EWMSC), San Fernando General Hospital (SFGH) and Port of Spain General Hospital (POSGH). Ethics approvals were granted by the University Campus Ethics Committee and the three Regional Health Authorities.

Clinical symptoms and laboratory diagnosis of nosocomial infections were noted and reviewed by the attending physician. Each week, prospective cases of nosocomial infections were reviewed from Doctor's notes in patient's dockets for any clinical signs of nosocomial infections three days (72hrs) following patient admission for all types of nosocomial infections, except for nosocomial bloodstream infections (BSI) of which was observed two days (48hrs) after admission.

A total of 2600 patient dockets for the duration of the research period were obtained at each of the research hospitals and reviewed for prospective cases of nosocomial infections. Codes were assigned for the nosocomial patient's names on the data collection sheets. In addition, the patient's names, their laboratory registration numbers, dates of samples collection and dates of sending samples to the laboratory by doctor or nurses were extremely useful to trace in the log book for their sample number, which helped to distinguish their respective nosocomial isolates from the others. The type of pathogen was confirmed by standard laboratory and biochemical tests. Samples were sent day one of patient's admission to confirm that the patients had no growth of organism on admission.

\section{Laboratory Diagnosis}

Manual antimicrobial susceptibility testing was performed using agar disk diffusion method for all bacterial isolates on Mueller Hinton agar as recommended by Clinical and Laboratory Standards [32]. Assessment of the infection control measures practiced at the research hospitals and mortality figures were reviewed and assessed from patient's dockets. In addition, total admission figures for all inpatients admitted on all researched wards were retrieved each month from the medical record unit at each of the regional hospitals, which was used to calculate the nosocomial rate. All samples were prepared and examined on at least three separate occasions and similar results were obtained. The antibiotics tested for resistance were amikacin (AK), amoxicillin/clavulanic acid (AMC), amoxicillin (AML), ampicillin (AMP), aztreonam (ATM), ceftazidime (CAZ), cefaclor (CEF), ciprofloxacin (CIP), clindamycin $(\mathrm{CN})$, cefuroxime (CXM), clindamycin (DA), ertapenem (ETP), nitrofurantoin (F), cefepime (FEP), gentamicin (GN), imipenem (IMP), levofloxacin (LEV), linezolid (LZD), meropenem (MEM), norfloxacin (NOR), piperacillin (PIP), trimethoprine/sulphamethazole (SXT), tetracycline (TE), tigercycline (TGC), tobramycin (TOB) and piperacillin/tazobactam (TZP).

\section{Data Analysis}

Descriptive statistics was used to calculate the sample size, incidence and rate of nosocomial infections and the percentage of nosocomial infections by age, months, research wards and nosocomial pathogens involved. Data related to patients and nosocomial infections were entered in a Microsoft ${ }^{\circledR}$ Access 2010 data bank and statistically processed using IBM SPSS $®$ Statistics (version 20). The statistical review of the study was done by a biomedical statistician.

\section{Sample Size Calculation:}

The sample size was calculated via a cluster sampling method by wards and patient's files [33]. The most practical sampling approach, and via the WHO and standard criteria for prospective nosocomial cases were selected from the defined population. Surveillance was conducted on a weekly basis for fifty-two (52) weeks, for prospective nosocomial cases and pathogen isolates that were obtained from the laboratory during the research period. The average confirmed number of nosocomial cases by WHO's definition was two per week. Therefore, via cluster sample size calculation equals number of wards multiplied by number of surveillance days and multiplied again by average number of patients with nosocomial infection confirmed during each week's surveillance. That is 5 wards $\times 52$ surveillance days $\times 2=520$ patients were expected to acquire nosocomial infection during the data collection period. Hence, 520 patients from clustered sample size calculation were expected to have been associated with nosocomial infections with confidence interval of $95 \%$ and relative precision of $10 \%$ within the research period. 


\section{Measurements}

In calculating the incidence of nosocomial infections in Trinidad and Tobago (2013 - 2014), the number of surveillance days (52) was multiplied by fifty (50) that was the average number of dockets reviewed each surveillance day from all the research wards: surgical, medical, paediatrics, ICU and Obstetrics \& Gynaecology. Therefore, fifty-two (52) multiplied by 50 equals 2600 cases reviewed at each research site. If this product is multiplied by three (3) equals 7800 files reviewed during the research period. Four hundred and fifty (450) nosocomial cases were recovered during the research period. The incidence of all three (3) regional hospitals was calculated by dividing number of confirmed cases over total number of reviewed files and multiplied by one hundred: $(450 / 7800) \times 100=5.8 \%$ and this was the incidence for inpatients associated with nosocomial infections at all major regional hospitals of Trinidad and Tobago during the specified period.

The incidence at site A was calculated by dividing the number of cases with nosocomial infection (265) divided by the total files reviewed (2600) and multiplied by 100 , which equaled to $10.2 \%$. Similarly, the incidence at site $\mathrm{B}$ was calculated by dividing the number of cases with nosocomial infection (63) divided by the total files reviewed (2600) and multiplied by 100 , which equaled to $2.4 \%$. The incidence at site $\mathrm{C}$ was calculated by dividing the number of hospital associated cases (122) divided by the total files reviewed (2600) and multiplied by 100 , which equaled to $4.7 \%$.

The nosocomial rate was calculated by dividing the number of nosocomial cases recovered during the research period, divided by the total number of patients admitted on the research wards and multiplied by 100 . The number of patients admitted on research wards for all three regional hospitals was 126,668 and the total number of nosocomial cases recovered for the research period was 450 . Hence, nosocomial rate for research period was; $450 / 126,668 \times 100=0.36 \% ; 3.6$ per 1000 patients. The nosocomial rate for research site A was $265 / 48057=0.55 \%(5.5$ per 1000 ), whereas the nosocomial rate for research site $\mathrm{B}$ was $63 / 39950=$ $0.16 \%$; 1.6 per 1000 and the nosocomial rate for research site $\mathrm{C}$ was $122 / 38,661=0.32 \% ; 3.2$ per 1000 patients.

Calculation of the antimicrobial susceptibility pattern rate (ASPR) for each pathogen strain was done as follows:

Number of antibiotics with susceptibility for a specific strain

$\mathrm{ASPR}=$

Total number of antibiotic tested for that specific strain x 100

\section{Result}

From out of 126668 admissions 450 nosocomial infections were recorded.

Table 1 reflects the frequency distribution of gender cases. Male patients (56\%) were predominantly more associated with nosocomial infections than female $(44 \%)$.

\begin{tabular}{llll}
\hline Gender & Number of Nosocomial Cases (n) & $\%$ & P-Value
\end{tabular}

\begin{tabular}{lcc}
\hline Male & 251 & 56 \\
1.960 & 199 & 4 \\
$\begin{array}{l}\text { Female } \\
1.960\end{array}$ & & \\
Total & 450 & 100
\end{tabular}

Table 1:- Frequency Distribution of Demographic Variables
Table 2 depicts frequency distribution of nosocomial infections by age groups. The age group 60 and over accounted for the highest percentage of nosocomial infections (26.7\%), followed by the age group 50-59 $(14.2 \%)$ and $40-49(11.8 \%)$ respectively.

\begin{tabular}{lcc}
\hline Age Group & Total NI (n) & $\%$ \\
\hline $0-9$ & 108 & 24 \\
$10-19$ & 16 & 3.6 \\
$20-29$ & 40 & 8.8 \\
$30-39$ & 49 & 10.9 \\
$40-49$ & 53 & 11.8 \\
$50-59$ & 64 & 14.2 \\
60 and above & 120 & 26.7 \\
Total & 450 & 100 \\
\hline
\end{tabular}

Table 2:- Age distribution of the Patients with Nosocomial Infections (NI)

Table 3 represents the distribution of nosocomial infection in each month of the research period. The month of August had the highest percentage of nosocomial infections $(12 \%)$, followed by the months of March (10.7\%) and November (10\%). The month of June (21) had the least percentage of nosocomial infections $(4.7 \%)$ and followed very closely by July $(4.9 \%)$.

\begin{tabular}{|c|c|c|}
\hline Month & $\begin{array}{c}\text { NI } \\
\text { frequency }\end{array}$ & $\%$ \\
\hline June & 21 & 4.7 \\
\hline July & 22 & 4.9 \\
\hline August & 54 & 12 \\
\hline September & 40 & 8.9 \\
\hline October & 40 & 8.9 \\
\hline November & 45 & 10 \\
\hline December & 29 & 6.4 \\
\hline January & 42 & 9.3 \\
\hline February & 44 & 9.8 \\
\hline March & 48 & 10.7 \\
\hline April & 30 & 6.7 \\
\hline May & 35 & 7.7 \\
\hline Total & 450 & 100 \\
\hline
\end{tabular}

Table 3:- Distribution of Nosocomial Infections (NI) in Trinidad and Tobago by Month 2013 - 2014

Table 4 depicts the frequency of microorganisms that were associated with nosocomial infections during the study period. Staphylococcus sp. (22.4\%) followed by Pseudomonas aeruginosa (12.7\%) accounted for the most frequent causative organism that were associated with nosocomial infections. These organisms were the most common multidrug resistant organisms producing many strains. Multiple pathogens (859) were mostly identified in each of the nosocomial cases of hospital-acquired infections. 


\begin{tabular}{lcc}
\hline Identified Organisms & Frequency & \% \\
\hline Staphylococcus sp. & 193 & 22.4 \\
Pseudomonas aeruginosa & 109 & 12.7 \\
Acinetobacter sp. & 101 & 11.8 \\
Klebsiella sp. & 100 & 11.6 \\
Enterobacter sp. & 74 & 8.6 \\
Enterococcus sp. & 64 & 7.4 \\
Escherichia coli & 58 & 6.8 \\
Candida albicans & 58 & 6.8 \\
Proteus sp. & 22 & 2.6 \\
Serratia sp. & 21 & 2.4 \\
Providencia stuartii & 14 & 1.6 \\
Stenotrophomonas maltophilia & 14 & 1.6 \\
Streptococcus sp. & 10 & 1.2 \\
Citrobacter koseri & 6 & 0.7 \\
Alcaligenes sp. & 6 & 0.7 \\
Morganella morgannii & 4 & 0.5 \\
Burkholderia (P) cepacia & 4 & 0.5 \\
Achromobacter xylosidans & 1 & 0.1 \\
Total & & \\
& &
\end{tabular}

Table 4:- Distribution of Identified Organisms associated with HAI in Trinidad and Tobago 2013 -2014

Footnote: HAI - Hospital Acquired Infections

Table 5 shows susceptibility patterns of Staphylococcus sp. These microorganisms showed 15 patterns of susceptibility, which indicate that 15 strains of Staphylococci exist as nosocomial pathogens in the hospital setting. At least, all strains were resistant to nine antibiotics including AML and $\mathrm{CN}$.

\section{Staphylococcus spp. (193/859) Antibiotics \\ RD TGC VA CXM E OX TOB SXT CN LZP CIP FOX AMC AML CEC GM CAZ TE}

\begin{tabular}{lllllllllllllllllll}
\hline a) & $\mathrm{S}$ & $\mathrm{S}$ & $\mathrm{S}$ & $\mathrm{R}$ & $\mathrm{R}$ & $\mathrm{R}$ & $\mathrm{R}$ & $\mathrm{R}$ & $\mathrm{R}$ & $\mathrm{R}$ & $\mathrm{R}$ & $\mathrm{R}$ & $\mathrm{R}$ & $\mathrm{R}$ & $\mathrm{R}$ & $\mathrm{R}$ & $\mathrm{R}$ & $\mathrm{R}$ \\
b) & $\mathrm{S}$ & $\mathrm{S}$ & $\mathrm{S}$ & $\mathrm{R}$ & $\mathrm{R}$ & $\mathrm{R}$ & $\mathrm{R}$ & $\mathrm{R}$ & $\mathrm{S}$ & $\mathrm{R}$ & $\mathrm{R}$ & $\mathrm{R}$ & $\mathrm{R}$ & $\mathrm{R}$ & $\mathrm{R}$ & $\mathrm{R}$ & $\mathrm{R}$ & $\mathrm{R}$ \\
$\mathrm{c})$ & $\mathrm{S}$ & $\mathrm{R}$ & $\mathrm{S}$ & $\mathrm{R}$ & $\mathrm{R}$ & $\mathrm{R}$ & $\mathrm{R}$ & $\mathrm{R}$ & $\mathrm{R}$ & $\mathrm{R}$ & $\mathrm{R}$ & $\mathrm{R}$ & $\mathrm{R}$ & $\mathrm{R}$ & $\mathrm{R}$ & $\mathrm{R}$ & $\mathrm{R}$ & $\mathrm{R}$ \\
$\mathrm{d})$ & $\mathrm{S}$ & $\mathrm{S}$ & $\mathrm{S}$ & $\mathrm{R}$ & $\mathrm{R}$ & $\mathrm{R}$ & $\mathrm{R}$ & $\mathrm{R}$ & $\mathrm{R}$ & $\mathrm{S}$ & $\mathrm{R}$ & $\mathrm{R}$ & $\mathrm{R}$ & $\mathrm{R}$ & $\mathrm{R}$ & $\mathrm{R}$ & $\mathrm{R}$ & $\mathrm{R}$ \\
$\mathrm{e})$ & $\mathrm{S}$ & $\mathrm{R}$ & $\mathrm{S}$ & $\mathrm{S}$ & $\mathrm{S}$ & $\mathrm{R}$ & $\mathrm{R}$ & $\mathrm{R}$ & $\mathrm{R}$ & $\mathrm{R}$ & $\mathrm{R}$ & $\mathrm{R}$ & $\mathrm{R}$ & $\mathrm{R}$ & $\mathrm{R}$ & $\mathrm{R}$ & $\mathrm{R}$ & $\mathrm{R}$ \\
$\mathrm{f}$ & $\mathrm{S}$ & $\mathrm{R}$ & $\mathrm{S}$ & $\mathrm{S}$ & $\mathrm{R}$ & $\mathrm{R}$ & $\mathrm{R}$ & $\mathrm{S}$ & $\mathrm{R}$ & $\mathrm{R}$ & $\mathrm{S}$ & $\mathrm{R}$ & $\mathrm{R}$ & $\mathrm{R}$ & $\mathrm{R}$ & $\mathrm{R}$ & $\mathrm{R}$ & $\mathrm{R}$ \\
$\mathrm{g})$ & $\mathrm{S}$ & $\mathrm{R}$ & $\mathrm{S}$ & $\mathrm{R}$ & $\mathrm{S}$ & $\mathrm{R}$ & $\mathrm{R}$ & $\mathrm{S}$ & $\mathrm{R}$ & $\mathrm{R}$ & $\mathrm{S}$ & $\mathrm{R}$ & $\mathrm{R}$ & $\mathrm{R}$ & $\mathrm{R}$ & $\mathrm{R}$ & $\mathrm{R}$ & $\mathrm{R}$ \\
h) & $\mathrm{R}$ & $\mathrm{R}$ & $\mathrm{R}$ & $\mathrm{R}$ & $\mathrm{S}$ & $\mathrm{S}$ & $\mathrm{S}$ & $\mathrm{S}$ & $\mathrm{R}$ & $\mathrm{R}$ & $\mathrm{R}$ & $\mathrm{R}$ & $\mathrm{R}$ & $\mathrm{R}$ & $\mathrm{R}$ & $\mathrm{R}$ & $\mathrm{R}$ & $\mathrm{R}$ \\
i) & $\mathrm{S}$ & $\mathrm{R}$ & $\mathrm{S}$ & $\mathrm{S}$ & $\mathrm{S}$ & $\mathrm{R}$ & $\mathrm{R}$ & $\mathrm{S}$ & $\mathrm{R}$ & $\mathrm{R}$ & $\mathrm{S}$ & $\mathrm{S}$ & $\mathrm{S}$ & $\mathrm{R}$ & $\mathrm{R}$ & $\mathrm{R}$ & $\mathrm{R}$ & $\mathrm{R}$ \\
j) & $\mathrm{S}$ & $\mathrm{S}$ & $\mathrm{S}$ & $\mathrm{R}$ & $\mathrm{R}$ & $\mathrm{R}$ & $\mathrm{R}$ & $\mathrm{R}$ & $\mathrm{R}$ & $\mathrm{R}$ & $\mathrm{R}$ & $\mathrm{R}$ & $\mathrm{R}$ & $\mathrm{R}$ & $\mathrm{R}$ & $\mathrm{R}$ & $\mathrm{R}$ & $\mathrm{R}$ \\
k) & $\mathrm{S}$ & $\mathrm{S}$ & $\mathrm{R}$ & $\mathrm{R}$ & $\mathrm{R}$ & $\mathrm{R}$ & $\mathrm{R}$ & $\mathrm{R}$ & $\mathrm{R}$ & $\mathrm{R}$ & $\mathrm{R}$ & $\mathrm{R}$ & $\mathrm{S}$ & $\mathrm{R}$ & $\mathrm{R}$ & $\mathrm{R}$ & $\mathrm{R}$ & $\mathrm{R}$ \\
1) & $\mathrm{S}$ & $\mathrm{S}$ & $\mathrm{S}$ & $\mathrm{R}$ & $\mathrm{R}$ & $\mathrm{R}$ & $\mathrm{R}$ & $\mathrm{R}$ & $\mathrm{R}$ & $\mathrm{R}$ & $\mathrm{R}$ & $\mathrm{R}$ & $\mathrm{R}$ & $\mathrm{R}$ & $\mathrm{R}$ & $\mathrm{R}$ & $\mathrm{R}$ & $\mathrm{R}$ \\
m) & $\mathrm{R}$ & $\mathrm{R}$ & $\mathrm{S}$ & $\mathrm{R}$ & $\mathrm{S}$ & $\mathrm{S}$ & $\mathrm{R}$ & $\mathrm{S}$ & $\mathrm{R}$ & $\mathrm{R}$ & $\mathrm{S}$ & $\mathrm{R}$ & $\mathrm{S}$ & $\mathrm{R}$ & $\mathrm{S}$ & $\mathrm{S}$ & $\mathrm{R}$ & $\mathrm{R}$ \\
$\mathrm{n}$ & $\mathrm{R}$ & $\mathrm{R}$ & $\mathrm{R}$ & $\mathrm{R}$ & $\mathrm{S}$ & $\mathrm{R}$ & $\mathrm{R}$ & $\mathrm{R}$ & $\mathrm{R}$ & $\mathrm{S}$ & $\mathrm{R}$ & $\mathrm{S}$ & $\mathrm{S}$ & $\mathrm{R}$ & $\mathrm{S}$ & $\mathrm{S}$ & $\mathrm{S}$ & $\mathrm{S}$ \\
o) & $\mathrm{S}$ & $\mathrm{R}$ & $\mathrm{S}$ & $\mathrm{R}$ & $\mathrm{S}$ & $\mathrm{R}$ & $\mathrm{R}$ & $\mathrm{S}$ & $\mathrm{R}$ & $\mathrm{R}$ & $\mathrm{S}$ & $\mathrm{S}$ & $\mathrm{S}$ & $\mathrm{R}$ & $\mathrm{R}$ & $\mathrm{S}$ & $\mathrm{R}$ & $\mathrm{S}$
\end{tabular}

Table 5: - Susceptibility Pattern of Staphylococcus spp. in Trinidad and Tobago 2013- 2014

Footnote: RD-Rifampicin, TGC- Tigercycline, VA-Vancomycin, CXM-Cefuroxime, E-Erythromycin, Ox-Oxacillin, TOB-Tobramycin, SXTTrimethoprine/Sulphamethazole, CN-Clindamycin, LZP-Linezolid, CIP-Ciprofloxacin, FOX-AMC-Amoxicillin/Clavulanic acid, AML-Amoxicillin, CEC-Cefaclor, GM-Gentamicin, CAZ-Ceftazidime, TE-Tetracycline.

Table 6 represents the susceptibility pattern of Pseudomonas aeruginosa organisms, which had four different patterns indicating that there are four different strains of the same organism. All strains were resistant to ATM,
ETP and TOB. On the other hand, the four strains were sensitive to TZP and CAZ. 
Pseudomonas aeroginosa (109/859)

Antibiotics

IPM TZP CAZ CIP CN GM ATM ETP ATM TOB

\begin{tabular}{lllllllllll}
\hline a) & $\mathrm{S}$ & $\mathrm{S}$ & $\mathrm{S}$ & $\mathrm{S}$ & $\mathrm{S}$ & $\mathrm{R}$ & $\mathrm{R}$ & $\mathrm{R}$ & $\mathrm{R}$ & $\mathrm{R}$ \\
b) & $\mathrm{S}$ & $\mathrm{S}$ & $\mathrm{S}$ & $\mathrm{S}$ & $\mathrm{R}$ & $\mathrm{S}$ & $\mathrm{R}$ & $\mathrm{R}$ & $\mathrm{S}$ & $\mathrm{R}$ \\
c) & $\mathrm{S}$ & $\mathrm{S}$ & $\mathrm{S}$ & $\mathrm{S}$ & $\mathrm{S}$ & $\mathrm{R}$ & $\mathrm{R}$ & $\mathrm{S}$ & $\mathrm{R}$ & $\mathrm{R}$ \\
d) & $\mathrm{R}$ & $\mathrm{S}$ & $\mathrm{S}$ & $\mathrm{S}$ & $\mathrm{R}$ & $\mathrm{S}$ & $\mathrm{S}$ & $\mathrm{R}$ & $\mathrm{R}$ & $\mathrm{S}$
\end{tabular}

Table 6: - Susceptibility Pattern of Pseudomonas aeroginosa in Trinidad and Tobago 2013- 2014
Footnote: IPM-Imipenem, TZP-Zosyn, CAZ-Ceftazidime, CIPCiprofloxacin, CN-Clindamycin, GM-Gentamicin, ATM-Aztreonam, ETP-Ertapenem, FEP-Cefepime and TOB-Tobramycin.

Table 7 depicts the antibiogram profile of Acinetobacter sp. indicating that five distinct strains of the same organism existed. The following antibiotics were observed to be most frequently resistant to Acinetobacter sp.: AML, AMC, ATM, CEC, ETP, CXM, GM and TE.

\section{Acinetobacter (101/859)}

Antibiotics

CAZ CIP ETP SXT TZP IMP TGC TOB AK CT TGC FEP CN AML AMC ATM CEC ETP CXM GM TE

\begin{tabular}{|c|c|c|c|c|c|c|c|c|c|c|c|c|c|c|c|c|c|c|c|}
\hline a) $\mathrm{S}$ & $S$ & $S$ & $S$ & $S$ & $S$ & $\mathrm{R}$ & $\mathrm{R}$ & $\mathrm{R}$ & $\mathrm{R}$ & $\mathrm{R}$ & $\mathrm{R}$ & $\mathrm{R}$ & $\mathrm{R}$ & $\mathrm{R}$ & $\mathrm{R}$ & $\mathrm{R}$ & $\mathrm{R}$ & $\mathrm{R}$ & $\mathrm{R} R$ \\
\hline b) $\mathrm{S}$ & $\mathrm{R}$ & $\mathrm{R}$ & $\mathrm{R}$ & $\mathrm{S}$ & $\mathrm{R}$ & $S$ & $\mathrm{~S}$ & $\mathrm{R}$ & $\mathrm{R}$ & $\mathrm{R}$ & $\mathrm{R}$ & $\mathrm{R}$ & $\mathrm{R}$ & $\mathrm{R}$ & $\mathrm{R}$ & $\mathrm{R}$ & $\mathrm{R}$ & $\mathrm{R}$ & $\mathrm{R}$ \\
\hline & $\mathrm{R}$ & $\mathrm{R}$ & $\mathrm{R}$ & $\mathrm{R}$ & $\mathrm{S}$ & $\mathrm{R}$ & $\mathrm{S}$ & $\mathrm{S}$ & $\mathrm{S}$ & $S$ & $\mathrm{R}$ & $\mathrm{R}$ & $\mathrm{R}$ & $\mathrm{R}$ & $\mathrm{R}$ & $\mathrm{R}$ & $\mathrm{R}$ & $\mathrm{R}$ & $\mathrm{R}$ \\
\hline $\mathrm{R}$ & $S$ & $\mathrm{R}$ & $\mathrm{S}$ & S & S & $\mathrm{R}$ & $\mathrm{R}$ & $\mathrm{R}$ & $\mathrm{R}$ & $\mathrm{R}$ & $\mathrm{S}$ & $\mathrm{S}$ & $\mathrm{R}$ & $\mathrm{R}$ & $\mathrm{R}$ & $\mathrm{R}$ & $\mathrm{R}$ & $\mathrm{R}$ & $\mathrm{R}$ \\
\hline$S$ & $S$ & $S$ & $S$ & $\mathrm{~S}$ & $\mathrm{R}$ & $\mathrm{R}$ & $\mathrm{R}$ & $\mathrm{R}$ & $\mathrm{R}$ & $\mathrm{R}$ & $\mathrm{R}$ & $\mathrm{R}$ & $\mathrm{R}$ & $\mathrm{R}$ & $\mathrm{R}$ & $\mathrm{R}$ & $\mathrm{R}$ & $\mathrm{R}$ & $\mathrm{R}$ \\
\hline
\end{tabular}

Table 7: Susceptibility Pattern of Acinetobacter in Trinidad and Tobago 2013- 2014

Footnote: CAZ-Ceftazidime, CIP-Ciprofloxacin, ETP-Ertapenem, SXT-Trimethoprine/Sulphamethaxole, TZP-Zosyn, IMP-Imipenem, TGC-

Tigercycline, TOB-Tobramycin, AK-Amikacin, CT-Colistin, FEP- Cefepime, CN-Clindamycin, AML-Amoxycillin, AMC-

Augmentin (Amoxicillin/Clavulanic acid), ATM-Aztreonam, CEC-Cefaclor, ETP-Ertapenem, CXM-Cefuroxime, GM-Gentamicin, TE-

Tetracycline.

Table 8 shows the susceptibility of Klebsiella sp. indicating that there are six (6) different strains of the same organism. The table depicted the presence of multidrug resistant strains of this pathogen.

\section{Klebsiella spp. (100/859)}

Antibiotics

CN IPM TZP AMC FEP CAZ CXM TOB CIP LEV TE ETP F NOR GM SXT AML CEC GM ATM TGC

\begin{tabular}{lcccccccccccccccccccc}
\hline a) $\mathrm{S}$ & $\mathrm{S}$ & $\mathrm{S}$ & $\mathrm{S}$ & $\mathrm{S}$ & $\mathrm{S}$ & $\mathrm{S}$ & $\mathrm{S}$ & $\mathrm{S}$ & $\mathrm{S}$ & $\mathrm{R}$ & $\mathrm{R}$ & $\mathrm{R}$ & $\mathrm{R}$ & $\mathrm{R}$ & $\mathrm{R}$ & $\mathrm{R}$ & $\mathrm{R}$ & $\mathrm{R}$ & $\mathrm{R}$ & $\mathrm{R}$ \\
b) R & $\mathrm{S}$ & $\mathrm{R}$ & $\mathrm{R}$ & $\mathrm{R}$ & $\mathrm{R}$ & $\mathrm{R}$ & $\mathrm{R}$ & $\mathrm{R}$ & $\mathrm{S}$ & $\mathrm{S}$ & $\mathrm{R}$ & $\mathrm{R}$ & $\mathrm{R}$ & $\mathrm{R}$ & $\mathrm{R}$ & $\mathrm{R}$ & $\mathrm{R}$ & $\mathrm{R}$ & $\mathrm{R}$ & $\mathrm{R}$ \\
c) R & $\mathrm{S}$ & $\mathrm{S}$ & $\mathrm{R}$ & $\mathrm{R}$ & $\mathrm{R}$ & $\mathrm{R}$ & $\mathrm{R}$ & $\mathrm{S}$ & $\mathrm{R}$ & $\mathrm{R}$ & $\mathrm{S}$ & $\mathrm{S}$ & $\mathrm{S}$ & $\mathrm{R}$ & $\mathrm{R}$ & $\mathrm{R}$ & $\mathrm{R}$ & $\mathrm{R}$ & $\mathrm{R}$ & $\mathrm{R}$ \\
d) R & $\mathrm{S}$ & $\mathrm{S}$ & $\mathrm{S}$ & $\mathrm{R}$ & $\mathrm{R}$ & $\mathrm{R}$ & $\mathrm{R}$ & $\mathrm{S}$ & $\mathrm{R}$ & $\mathrm{R}$ & $\mathrm{S}$ & $\mathrm{R}$ & $\mathrm{S}$ & $\mathrm{R}$ & $\mathrm{S}$ & $\mathrm{R}$ & $\mathrm{R}$ & $\mathrm{S}$ & $\mathrm{R}$ & $\mathrm{R}$ \\
e) S & $\mathrm{S}$ & $\mathrm{S}$ & $\mathrm{R}$ & $\mathrm{R}$ & $\mathrm{R}$ & $\mathrm{R}$ & $\mathrm{R}$ & $\mathrm{S}$ & $\mathrm{R}$ & $\mathrm{R}$ & $\mathrm{R}$ & $\mathrm{R}$ & $\mathrm{R}$ & $\mathrm{R}$ & $\mathrm{R}$ & $\mathrm{R}$ & $\mathrm{R}$ & $\mathrm{R}$ & $\mathrm{R}$ & $\mathrm{R}$ \\
f) S & $\mathrm{R}$ & $\mathrm{S}$ & $\mathrm{S}$ & $\mathrm{S}$ & $\mathrm{S}$ & $\mathrm{R}$ & $\mathrm{R}$ & $\mathrm{S}$ & $\mathrm{R}$ & $\mathrm{R}$ & $\mathrm{R}$ & $\mathrm{R}$ & $\mathrm{R}$ & $\mathrm{R}$ & $\mathrm{R}$ & $\mathrm{R}$ & $\mathrm{S}$ & $\mathrm{R}$ & $\mathrm{S}$ & $\mathrm{S}$
\end{tabular}

Table 8: -Susceptibility Pattern of Klebsiella spp. in Trinidad and Tobago 2013- 2014

Footnote: CN-Clindamycin, IPM-Imipenem, TZP-Zosyn, AMC-Augmentin (Amoxicillin/Clavulanic acid), FEP-Cefepime, CAZ-Ceftazidime, CXM-Cefuroxime, TOB-Tobramycin, CIP-Ciprofloxacin, LEV-Levofloxacin, TE-Tetracycline, ETP-Ertapenem, F-Nitrofurantoin, NOR-

Norfloxacin, GM-Gentamicin, SXT-Trimethoprine/

Sulphamethaxole, AML-Amoxycillin, CEC-Cefaclor, ATM-Aztreonam, TGC-

Tigercycline.

Table 9 represents the susceptibility of Enterobacter sp. indicating that five different strains of the same organism existed. This microorganism's strains were resistant to AK, FEP and TE; and mostly susceptible to CAZ, CIP and TZP. 


\begin{tabular}{|c|c|c|c|c|c|c|c|c|c|c|c|c|c|}
\hline \multicolumn{14}{|c|}{$\begin{array}{c}\text { Enterobacter }(74 / 859) \\
\text { Antibiotics }\end{array}$} \\
\hline a) $\mathrm{S}$ & $S$ & $S$ & $S$ & $S$ & $\mathrm{R}$ & $S$ & R & $\mathrm{R}$ & R & R & $\mathrm{R}$ & $\mathrm{R}$ & $\mathrm{R}$ \\
\hline b) $\mathrm{S}$ & $\mathrm{S}$ & $S$ & $S$ & $S$ & $S$ & $S$ & $\mathrm{R}$ & $\mathrm{R}$ & $\mathrm{R}$ & $\mathrm{R}$ & $\mathrm{R}$ & $\mathrm{R}$ & $\mathrm{R}$ \\
\hline c) $\mathrm{S}$ & $\mathrm{S}$ & $S$ & $S$ & $S$ & $\mathrm{R}$ & $\mathrm{S}$ & $\mathrm{S}$ & $S$ & $\mathrm{~S}$ & $\mathrm{R}$ & $\mathrm{R}$ & $\mathrm{R}$ & $\mathrm{R}$ \\
\hline d) $\mathrm{S}$ & $\mathrm{S}$ & $\mathrm{R}$ & $\mathrm{R}$ & $S$ & $S$ & $\mathrm{R}$ & $S$ & $\mathrm{R}$ & $\mathrm{R}$ & $S$ & $\mathrm{~S}$ & $\mathrm{R}$ & $\mathrm{R}$ \\
\hline e) $\mathrm{S}$ & $S$ & $\mathrm{R}$ & $\mathrm{R}$ & $S$ & $\mathrm{R}$ & $\mathrm{R}$ & $\mathrm{R}$ & $S$ & $\mathrm{R}$ & $\mathrm{R}$ & $S$ & $S$ & $\mathrm{~S}$ \\
\hline
\end{tabular}

Table 9: - Susceptibility Pattern of Enterobacter in Trinidad and Tobago 2013- 2014

Footnote: CAZ-Ceftazidime, CIP-Ciprofloxacin, ETP-Ertapenem, SXT-Trimethoprine/Sulphamethaxole, TZP-Zosyn, TOB-Tobramycin, IMP-Imipenem, GM-Gentamicin, ATM-Aztreonam, CXM-Cefuroxime, AK-Amikacin, FEP-Cefepime,

$\mathrm{CN}-$ Clindamycin, TE-Tetracycline.

Table 10 features the susceptibility of Enterococcus sp. This pathogen had three different susceptibility patterns inferring that three different strains of the same organism existed.

Enterococcus spp. (64/859)

Antibiotic

CEC CXM AMC ETP TZP IPM CAZ CIP CT CN AK

\begin{tabular}{llllllllllll}
\hline a) & $\mathrm{S}$ & $\mathrm{S}$ & $\mathrm{S}$ & $\mathrm{R}$ & $\mathrm{R}$ & $\mathrm{R}$ & $\mathrm{R}$ & $\mathrm{R}$ & $\mathrm{R}$ & $\mathrm{R}$ & $\mathrm{R}$ \\
b) & $\mathrm{R}$ & $\mathrm{R}$ & $\mathrm{R}$ & $\mathrm{S}$ & $\mathrm{S}$ & $\mathrm{S}$ & $\mathrm{R}$ & $\mathrm{R}$ & $\mathrm{R}$ & $\mathrm{R}$ & $\mathrm{R}$ \\
c) & $\mathrm{R}$ & $\mathrm{S}$ & $\mathrm{R}$ & $\mathrm{R}$ & $\mathrm{R}$ & $\mathrm{S}$ & $\mathrm{S}$ & $\mathrm{S}$ & $\mathrm{S}$ & $\mathrm{S}$ & $\mathrm{S}$
\end{tabular}

Table 10: - Susceptibility Pattern of Enterococcus spp. in Trinidad and Tobago 2013- 2014

Footnote: CEC-Cefaclor, CXM-Cefuroxime, AMC-Augmentin (Amoxicillin/Clavulanic acid), ETP-Ertapenem, TZP-Zosyn, IPMImipenem, CAZ-Ceftazidime, CIP-Ciprofloxacin, CT-Colistin, CNClindamycin, AK-Amikacin.

Table 11 depicts three susceptibility patterns of Escherichia coli inferring that there is three different strains of the same organism. Two of the strains were multi-resistant to ten or more antibiotics.

\section{Escherichia coli (58/859)}

Antibiotics

CF CN SXT AMP AMC AML ATM CEC CAZ CIP E ETP GM IPM TZP FEP TGC

\begin{tabular}{llllllllllllllllll}
\hline a) & $\mathrm{S}$ & $\mathrm{S}$ & $\mathrm{S}$ & $\mathrm{S}$ & $\mathrm{S}$ & $\mathrm{R}$ & $\mathrm{R}$ & $\mathrm{R}$ & $\mathrm{R}$ & $\mathrm{R}$ & $\mathrm{R}$ & $\mathrm{R}$ & $\mathrm{R}$ & $\mathrm{R}$ & $\mathrm{R}$ & $\mathrm{R}$ & $\mathrm{R}$ \\
b) & $\mathrm{R}$ & $\mathrm{R}$ & $\mathrm{S}$ & $\mathrm{R}$ & $\mathrm{S}$ & $\mathrm{S}$ & $\mathrm{S}$ & $\mathrm{S}$ & $\mathrm{S}$ & $\mathrm{S}$ & $\mathrm{S}$ & $\mathrm{S}$ & $\mathrm{S}$ & $\mathrm{S}$ & $\mathrm{S}$ & $\mathrm{R}$ & $\mathrm{R}$ \\
c) & $\mathrm{R}$ & $\mathrm{R}$ & $\mathrm{R}$ & $\mathrm{R}$ & $\mathrm{R}$ & $\mathrm{S}$ & $\mathrm{S}$ & $\mathrm{R}$ & $\mathrm{R}$ & $\mathrm{R}$ & $\mathrm{R}$ & $\mathrm{R}$ & $\mathrm{R}$ & $\mathrm{R}$ & $\mathrm{S}$ & $\mathrm{S}$ & $\mathrm{S}$
\end{tabular}

Table 11:Susceptibility Pattern of Escherichia coli in Trinidad and Tobago 2013- 2014
Footnote: $\quad \mathrm{CF} / \mathrm{KF}-$ Cephalothin, $\quad \mathrm{CN}$-Clindamycin, SXTTrimethoprine/Sulphamethaxole, AMP-Ampicillin, AMC-Augmentin (Amoxillin/Clavulanic acid), AML-Amoxycillin, ATM-Aztreonam, CEC-Cefaclor, CAZ-Ceftazidime, CIP-Ciprofloxacin, E-Erythromycin, ETP-Ertapenem, GM-Gentamicin, IPM-Imipenem, TZP-Zosyn, FEP Cefepime, TGC-Tigercycline.

Table 12 shows two susceptibility patterns for Proteus sp. indicating that two strains of the same organism existed. The two strains were resistant to $\mathrm{F}$ and SXT and sensitive to TZP.

\section{Antibiotic Profile of Proteus spp. (22/859) \\ Antibiotic \\ CAZ CIP CN TZP AMC CEC GM IPM NOR F SXT}

$\begin{array}{llllllllllll}\text { a) } & \mathrm{S} & \mathrm{S} & \mathrm{S} & \mathrm{S} & \mathrm{R} & \mathrm{R} & \mathrm{R} & \mathrm{R} & \mathrm{R} & \mathrm{R} & \mathrm{R} \\ \text { b) } & \mathrm{R} & \mathrm{R} & \mathrm{R} & \mathrm{S} & \mathrm{S} & \mathrm{S} & \mathrm{S} & \mathrm{S} & \mathrm{S} & \mathrm{R} & \mathrm{R}\end{array}$

Table 12: Susceptibility Pattern of Proteus spp. in Trinidad and Tobago 2013- 2014

Footnote: CAZ-Ceftazidime, CIP-Ciprofloxacin, CN-Clindamycin, TZP-Zosyn, AMC-Augmentin (Amoxicillin/Clavulanic acid), CECCefaclor, GM-Gentamicin, IPM-Imipenem, NOR-Norfloxacin, F/MNitrofurantoin, SXT-Trimethoprine/Sulphamethaxole.

Table 13 represents seven (7) distinct susceptibility patterns of Serratia marcescens, indicating that seven (7) different strains of the same organism existed. All strains were resistant to AML, AMC and CEC. Most strains were sensitive to ATM, CAZ, TZP and CIP. Six of Seven (6/7) strains were resistant to TGC, CEC, CT, LEV, IMP and PIP.

Serratia spp. (21/859) Antibiotic

ATM CAZ FEP CIP CN TZP TE AK TOB AML AMC CEC SXT GM TGC CT LEV IMP PIP ETP

$\begin{array}{lllllllllllllllllllll}\text { a) } & \mathrm{S} & \mathrm{S} & \mathrm{S} & \mathrm{S} & \mathrm{S} & \mathrm{S} & \mathrm{S} & \mathrm{R} & \mathrm{R} & \mathrm{R} & \mathrm{R} & \mathrm{R} & \mathrm{R} & \mathrm{R} & \mathrm{R} & \mathrm{R} & \mathrm{R} & \mathrm{R} & \mathrm{R} & \mathrm{R} \\ \text { b) } & \mathrm{S} & \mathrm{S} & \mathrm{S} & \mathrm{S} & \mathrm{R} & \mathrm{S} & \mathrm{S} & \mathrm{S} & \mathrm{S} & \mathrm{R} & \mathrm{R} & \mathrm{R} & \mathrm{R} & \mathrm{S} & \mathrm{R} & \mathrm{R} & \mathrm{R} & \mathrm{R} & \mathrm{R} & \mathrm{R} \\ \text { c) } & \mathrm{S} & \mathrm{S} & \mathrm{R} & \mathrm{S} & \mathrm{R} & \mathrm{S} & \mathrm{R} & \mathrm{R} & \mathrm{R} & \mathrm{R} & \mathrm{R} & \mathrm{R} & \mathrm{S} & \mathrm{S} & \mathrm{R} & \mathrm{R} & \mathrm{R} & \mathrm{R} & \mathrm{R} & \mathrm{R} \\ \text { d) } & \mathrm{S} & \mathrm{R} & \mathrm{R} & \mathrm{R} & \mathrm{R} & \mathrm{R} & \mathrm{R} & \mathrm{S} & \mathrm{R} & \mathrm{R} & \mathrm{R} & \mathrm{R} & \mathrm{R} & \mathrm{R} & \mathrm{R} & \mathrm{R} & \mathrm{R} & \mathrm{R} & \mathrm{R} & \mathrm{R} \\ \text { e) } & \mathrm{S} & \mathrm{S} & \mathrm{S} & \mathrm{S} & \mathrm{R} & \mathrm{S} & \mathrm{S} & \mathrm{R} & \mathrm{R} & \mathrm{R} & \mathrm{R} & \mathrm{R} & \mathrm{R} & \mathrm{S} & \mathrm{S} & \mathrm{R} & \mathrm{R} & \mathrm{R} & \mathrm{R} & \mathrm{R} \\ \mathrm{f} & \mathrm{S} & \mathrm{S} & \mathrm{R} & \mathrm{S} & \mathrm{R} & \mathrm{S} & \mathrm{R} & \mathrm{R} & \mathrm{R} & \mathrm{R} & \mathrm{R} & \mathrm{R} & \mathrm{S} & \mathrm{S} & \mathrm{R} & \mathrm{S} & \mathrm{R} & \mathrm{R} & \mathrm{R} & \mathrm{S} \\ \text { g) } & \mathrm{R} & \mathrm{R} & \mathrm{S} & \mathrm{R} & \mathrm{R} & \mathrm{R} & \mathrm{R} & \mathrm{R} & \mathrm{S} & \mathrm{R} & \mathrm{R} & \mathrm{R} & \mathrm{S} & \mathrm{R} & \mathrm{R} & \mathrm{R} & \mathrm{S} & \mathrm{S} & \mathrm{S} & \mathrm{R}\end{array}$

Table 13: - Susceptibility Pattern of Serratia spp. in Trinidad and Tobago 2013- 2014 
Footnote: ATM-Aztreonam, CAZ-Ceftazidime, FEP-Cefepime, CIP-Ciprofloxacin, CN-Clindamycin, TZP(PIP/TAXO)-Zosyn, TE-Tetracycline, AK-Amikacin, TOB-Tobramycin, AML-Amoxycillin, AMC-Augmentin (Amoxicillin/Clavulanic acid), CEC-Cefaclor, SXTTrimethoprine/Sulphamethaxole, GM-Gentamicin, TGC-Tigercycline, CT-Colistin, LEV-Levofloxacin, IMP-Imipenem, ETP-Ertapenem.

Table 14 depicts one susceptibility pattern for Providencia stuartii sp. indicating that one strain of the organism existed. This pathogen was sensitive to the three antibiotics (AK, TGC and MEM) tested.

\begin{tabular}{llll}
\hline & AK & TGC & MEM \\
\hline a) & $\mathrm{S}$ & $\mathrm{S}$ & $\mathrm{S}$ \\
\hline
\end{tabular}

Table 14: Susceptibility Pattern of Providencia Stuartii (14/859) in Trinidad and Tobago 2013- 2014

Footnote: AK-Amikacin, TGC-Tigercycline, MEM-Meropenem

Table 15 features two (2) different susceptibility patterns of the Stenotrophomonas maltophilia inferring that two (2) different strains of the same organism existed.

\begin{tabular}{rrrrrrr}
\hline & AK & TGC & MEM & CAZ & LEV & SXT \\
\hline a) & $\mathrm{S}$ & $\mathrm{S}$ & $\mathrm{S}$ & $\mathrm{R}$ & $\mathrm{R}$ & $\mathrm{R}$ \\
b) & $\mathrm{R}$ & $\mathrm{R}$ & $\mathrm{R}$ & $\mathrm{S}$ & $\mathrm{S}$ & $\mathrm{S}$ \\
\hline
\end{tabular}

Table 15: Susceptibility Pattern of Stenotrophomonas maltophilia (14/859) in Trinidad and Tobago 2013- 2014.

Footnote: AK-Amikacin, TGC-Tigercycline, MEM-Meropenem, CAZCeftazidime, LEV-Levofloxacin, SXT-Trimethoprine/Sulphamethaxole.

Table 16 shows one susceptibility pattern for Streptococcus sp. inferring that one strain of the organism was present.

GM TZP MEM CAZ FEP

a) $\mathrm{S} \quad \mathrm{S} \quad \mathrm{S} \quad \mathrm{S} \quad \mathrm{S}$

Table 16:Susceptibility Pattern of Streptococcus spp. (10/859) in Trinidad and Tobago 2013- 2014.

Footnote: GM-Gentamicin, TZP-Zosyn, MEM-Meropenem, CAZCeftazidime, FEP-Cefepime.

Table 17 depicts two (2) susceptibility patterns of the Citrobacter koseri indicating that there are two (2) strains of this organism circulating at regional hospital in Trinidad and Tobago. Both strains were resistant to AMP and TE and sensitive to TZP and CIP.

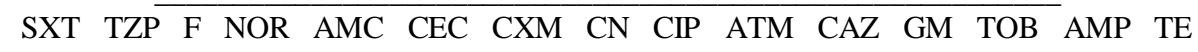

\begin{tabular}{llllllllllllllll}
\cline { 2 - 4 } & $\mathrm{S}$ & $\mathrm{S}$ & $\mathrm{S}$ & $\mathrm{S}$ & $\mathrm{S}$ & $\mathrm{S}$ & $\mathrm{S}$ & $\mathrm{S}$ & $\mathrm{S}$ & $\mathrm{R}$ & $\mathrm{R}$ & $\mathrm{R}$ & $\mathrm{R}$ & $\mathrm{R}$ & $\mathrm{R}$ \\
b) & $\mathrm{R}$ & $\mathrm{S}$ & $\mathrm{R}$ & $\mathrm{R}$ & $\mathrm{R}$ & $\mathrm{R}$ & $\mathrm{R}$ & $\mathrm{R}$ & $\mathrm{S}$ & $\mathrm{S}$ & $\mathrm{S}$ & $\mathrm{S}$ & $\mathrm{S}$ & $\mathrm{R}$ & $\mathrm{R}$
\end{tabular}

\section{Table 17: Susceptibility Pattern of Citrobacter koseri (6/859) in Trinidad and Tobago 2013- 2014.}

Footnote: SXT-Trimethoprine/Sulphamethaxole, TZP-Zosyn, Nitrofurantoin, NOR-Norfloxacin, AMC-Augmentin (Amoxicillin/Clavulanicacid), CEC-Cefaclor, CXM Cefuroxime, CNClindamycin, CIP-Ciprofloxacin, ATM-Aztreonam, CAZ-Ceftazidime, GM-Gentamicin, TOB-

Tobramycin, AMP-Ampicillin, TE-Tetracycline

Table 18 features two (2) susceptibility patterns of Alcaligenes sp. indicating that two strains of the same organism were present. This pathogen was sensitive to CAZ, CIP and TZP.

\section{CAZ CIP TZP SXT TOB CN TE}

\begin{tabular}{llllllll}
\hline a) & $\mathrm{S}$ & $\mathrm{S}$ & $\mathrm{S}$ & $\mathrm{S}$ & $\mathrm{R}$ & $\mathrm{R}$ & $\mathrm{R}$ \\
b) & $\mathrm{S}$ & $\mathrm{S}$ & $\mathrm{S}$ & $\mathrm{R}$ & $\mathrm{S}$ & $\mathrm{S}$ & $\mathrm{S}$
\end{tabular}

b) $\begin{array}{lllllll}\mathrm{S} & \mathrm{S} & \mathrm{S} & \mathrm{R} & \mathrm{S} & \mathrm{S} & \mathrm{S}\end{array}$

Table 18: Susceptibility Pattern of Alcaligenes (6/859) in Trinidad and Tobago 2013- 2014.

Footnote: CAZ- Ceftazidime, CIP-Ciprofloxacin, TZP-Zosyn, SXTTrimethoprine/Sulphamethaxole, TOB-Tobramycin, CN-Clindamycin, TE-Tetracycline.
Table 19 represents one (1) susceptible pattern inferring that only one (1) strain of the Morganella morgannii organism was present. This strain was sensitive to all antibiotics tested.

\section{ATM FEP CIP GM IPM TZP}

a) $\begin{array}{llllll}\mathrm{S} & \mathrm{S} & \mathrm{S} & \mathrm{S} & \mathrm{S} & \mathrm{S}\end{array}$

Table 19: Susceptibility Pattern of Morganella morgannii (4/859) in Trinidad and Tobago 2013- 2014.

Footnote: ATM- Aztreonam, FEP-Cefepime, CIP-Ciprofloxacin, GMGentamicin, IPM-Imipenem and TZP-Zosyn. 
Table 20 depicts one (1) susceptibility pattern inferring that Burkholderia (P) cepacia has only one strain present at the research sites. It was resistant to AK, CIP, GM and TE and sensitive to many antibiotics.

\section{CAZ FEP TZP TGC TOB SXT AK CIP GM TE}

\begin{tabular}{lllllllllll}
\hline a) & $\mathrm{S}$ & $\mathrm{S}$ & $\mathrm{S}$ & $\mathrm{S}$ & $\mathrm{S}$ & $\mathrm{S}$ & $\mathrm{R}$ & $\mathrm{R}$ & $\mathrm{R}$ & $\mathrm{R}$
\end{tabular}

Table 20:Susceptibility Pattern of Burkholderia $(P)$ cepacia (4/859) in Trinidad and Tobago 2013- 2014.

Footnote: CAZ-Ceftazidime, FEP-Cefepime, TZP-Zosyn, TGCTigercycline, TOB-Tobramycin, SXT-Trimethoprine/Sulphamethaxole, AK-Amikacin, CIP-Ciprofloxacin, GM-Gentamicin, TE-Tetracycline.

Table 21 shows the simplified criteria for surveillance of nosocomial infections. It serves as a practical guide for diagnosis and management of different types of nosocomial infections [61].

\begin{tabular}{|l|l|}
\hline \multicolumn{1}{|c|}{$\begin{array}{c}\text { Type of } \\
\text { Nosocomial } \\
\text { Infection }\end{array}$} & \multicolumn{1}{|c|}{ Simplified Criteria Infection } \\
\hline $\begin{array}{l}\text { Surgical site } \\
\text { infection }\end{array}$ & $\begin{array}{l}\text { Any purulent discharge, abscess, or spreading } \\
\text { cellulitis at the surgical site during the month after } \\
\text { the operation }\end{array}$ \\
\hline $\begin{array}{l}\text { Urinary } \\
\text { infection }\end{array}$ & $\begin{array}{l}\text { Positive urine culture (1 or 2 species) with at least } \\
105 \text { bacteria/ml, with or without clinical symptoms }\end{array}$ \\
\hline $\begin{array}{l}\text { Respiratory } \\
\text { infection }\end{array}$ & $\begin{array}{l}\text { Respiratory symptoms with at least two of the } \\
\text { following signs appearing during hospitalization: } \\
\text { - cough - purulent sputum - new infiltrate on } \\
\text { chest radiograph consistent with infection Vascular } \\
\text { catheter Inflammation Lymphangitis or infection }\end{array}$ \\
\hline
\end{tabular}

\begin{tabular}{|l|l|}
\hline & $\begin{array}{l}\text { with purulent discharge at the insertion site of the } \\
\text { catheter }\end{array}$ \\
\hline Septicaemia & $\begin{array}{l}\text { Fever or rigors and at least one positive } \\
\text { blood culture }\end{array}$ \\
\hline
\end{tabular}

Table 21: Simplified Criteria for Surveillance of Nosocomial Infections by World Health Organization (WHO).

Table 22 features the different antimicrobial susceptibility pattern rates (ASPR) for each pathogen. Strains of Staphylococcus sp. showed low susceptibility pattern that range between $5.6 \%$ and $50 \%$ indicating the presence of multidrug resistance. Most strains isolated have an ASPR below 50\% and this is worrisome for the management of hospitalacquired infections. This stresses the necessity of good hygiene practices and caution in the misuse of broad-spectrum antibiotics and new generation antimicrobials for the treatment of minor bacterial infections. All strains of Acinetobacter sp. (15.0\%-40.0\%), Serratia marcescens (10.0\%-45.0\%) and Klebsiella sp. (10.0\%-45.0\%), as shown in the table have an ASPR below $50 \%$, which is the cut-off point established based on the availability of antimicrobials. On the other hand, Providencia stuartii, Streptococcus sp., Morganella morganii and Burkholderia cepacia were susceptible to the $100 \%$ of antibiotics used in the antibiogram. It is good news that these pathogens have yet not develop antibiotic resistance, since they have started becoming more prevalent in the hospital facilities. Multidrug resistant strains of Providencia stuartii [77], Streptococcus sp. [78], Morganella morganii [79] and Burkholderia cepacia [80] have been reported. Antibiotic resistance can be acquired by horizontal transfer of a resistance gene or mutation and generally an acquired mechanism results in a predictable increase in phenotypic resistance [78]. A surveillance programme is in place at the 3 regional hospitals to monitor susceptibility patterns of these microorganisms, to delay antibiotic resistance in the future. The fact that antibiotic resistance has not been developed for these four (4) pathogens represents a success of the institutional antimicrobial stewardship.

\begin{tabular}{|c|c|c|c|}
\hline $\begin{array}{l}\text { Nosocomial Microorganisms } \\
\quad \mathrm{ASPR} \leq 50 \%\end{array}$ & \# of strains & $\begin{array}{l}\text { \# of strains } \\
\text { ASPR } \geq 51\end{array}$ & $\begin{array}{l}\text { ASPR } \\
(\%)\end{array}$ \\
\hline Citrobacter koseri & 1 & 1 & $40.0-60.0$ \\
\hline Stenotrophomonas maltophilia & 2 & 0 & 50.0 \\
\hline Alcaligenes sp. & 0 & 2 & $57.1-85.7$ \\
\hline Providencia stuartii & 0 & 1 & 100 \\
\hline Streptococcus sp. & 0 & 1 & 100 \\
\hline Morganella morgannii & 0 & 1 & 100 \\
\hline Burkholderia cepacia & 0 & 1 & 100 \\
\hline
\end{tabular}

Table 22: Antimicrobial Susceptibility Pattern Rates (ASPR) for Nosocomial Pathogens Isolated at Three (3) Regional Hospitals in Trinidad and Tobago during the Period June $1^{\text {st }}, 2013$ to May $31^{\text {st }}, 2014$.

\section{Discussion}

In Trinidad and Tobago information on nosocomial infections are lacking. Orrett, 2002 documented that 7,158 hospital acquired infections were observed from 72,532 patients. High nosocomial rates were observed in the intensive care unit (ICU) (67/100), urology (30/100), neurosurgery (29.5/100) and new-born nursery (28.4/100) wards. Nosocomial urinary tract infections (4.1/100) accounted for the majority of nosocomial infections (42\%), followed by post-operative nosocomial wound infections (26.8\%) having a nosocomial rate of 2.6/100 [9]. The researcher observed from the research that the incidence of nosocomial infections was $5.8 \%$ which was lesser when compared to Benin, another developing country, whose incidence was $19.1 \%[6,26]$. There were other nations (developing countries) with higher incidences when compared to the researcher's findings [27].

The age of patients who had nosocomial infections ranged from eight (8) days old to ninety- six (96) years old. Neonates were more susceptible of acquiring nosocomial infections $(108 / 450 ; 24 \%)$ and this was due to 
their weak immune system. It was reported in the literature that children, who developed nosocomial infections had a three-fold increase in hospital stay ( 27 days versus 9 days, $\mathrm{p}<0.001$ ) compared to those who did not [14]. An increased expression of cytokine genes (IL1B and IL10) was observed in patients, who developed nosocomial infections, in addition to a pro-apoptosis pattern due to a lower expression of BCL2. CD3D, a key TCR co-factor that was found significantly downmodulated in children, which developed nosocomial infections [34]. Frequency of nosocomial cases amongst elderly inpatients were higher than other age group, and this was supported by several studies [35-42], which reported that it may be due to risk factors such as impaired immunity, chronic diseases, medications, malnutrition, and functional impairments, among others.

This study showed that surgical wards had the highest nosocomial infection rate $(33.8 \%)$. But in a surgical emergency department was verified or suspected aspiration as the most important risk factor for the development of nosocomial infection [43] and we agreed with that. Another research showed that the highest surgical site infection (SSI) incidence was after dirty surgery and was $17.8 \%$ (102 out of 574) in patients living in high human development index (HDI) countries. Patients in middle-HDI countries had an incidence of $31.4 \%$ (74 out of 236 ), and the poorest countries had an incidence of approximately $40 \%$ (72 out of 181) [44]. The use of prophylactic antibiotics for the prevention of SSI has been cited but it may not be available for third world countries [45]. This data indicate that poverty and hospital environment are also risk factors for nosocomial infections and we stressed the need of measures to control and prevent infections from pathogens that live in the hospital microbiota. In addition, a high incidence of nosocomial infection $(28.15 \%)$ was seen in intensive care units in a study carried out in Ethiopia, where the risk of nosocomial infection was found to be higher in patients with chest tube, on mechanical ventilation or underlying diseases $[2,46]$.

Skin and soft tissue infection was the most frequent type of nosocomial infection shown in this study, accounting for $37.3 \%$ (168 out of 450$)$. It was reported that the most frequent clinical syndromes in 174 infection episodes in patients $\geq 65$ years old were osteoarticular (40\%) and skin and soft-tissue infections $(30 \%)$ in a research aiming to study the frequency distribution of syndromes in the elderly, where bacteraemia was found in $46 \%$ of the skin and soft tissue nosocomial infections [47]. Despite the considerable effort devoted to observing each ward, it is of vital importance that key focus be placed on the surgical, medical and paediatric facilities, where the nosocomial cases were highest. Another aspect of great interest is to put special attention in sterilizing surgical tools, frequent hand washing and changing of gloves as often as possible. Reichmann DE \& Greenberg JA, 2009 reported that patient skin preparation in the operating room, usage of chlorhexidine- based preparations, pre-operative hand/forearm antisepsis, hair removal, etc. should be considered for safe procedures, when carried out on the surgical wards to reduce the nosocomial rate [48].

In this investigation Staphylococcus sp. (22.4\%; 193/859) and Pseudomonas aeruginosa $(12.6 \% ; 109 / 859)$ organisms were one of the principal gram-positive and gram-negative nosocomial pathogens respectively connected with hospital acquired infections during the research period. The cumulative occurrence of multi-drug-resistant Staphylococcus sp. and Pseudomonas aeruginosa strains were alarming as effective antibiotic choices were severely limited. Other frequently associated multidrug resistant nosocomial organisms included Acinetobacter sp. (11.8\%; 101/859) and Klebsiella sp. (11.6\%; 100/859). In addition, Klebsiella and Staphylococcus sp. were the most frequently observed causative organisms in nosocomial bloodstream infections in our investigation. Several scientists studied nosocomial bloodstream infections caused by Pseudomonas sp. in new-borns and concluded that they were very frequent in neonates and a cause of bacteraemia and mortality, which may be due to low birth weight, underlying disorders and invasive procedures as predisposing factors [49-50]. Other authors published the most commonly isolated microorganisms in new-born ICU in another research. It was Klebsiella sp. (39.6\%) followed by Pseudomonas aeruginosa $(11.3 \%)$ and coagulase-negative staphylococci (9.4\%) [7-8, 51]. Our results along with what has been previously reported in the literature suggest that gram-negative bacteria (especially P. aeruginosa and Klebsiella sp.) play an important role in the pathogenesis of nosocomial infections in neonates admitted in ICU. The neonate's immune system is not mature enough to fight off these infections caused by these organisms and thus linked to their virulence factors create a perfect scenario for bacteraemia, septic shock and other complications $[4,15]$.

Nosocomial skin and soft tissue infections (SSTI) and bloodstream infections (BSI) were the most common types of nosocomial infections with occurrences of $37.3 \%$ and $28.4 \%$ respectively, but their incidence can be dropped by good hygiene practices, and this has been observed by other authors [72-75]. These infections were commonly transmitted via direct contact. Respiratory tract infections (RTI) and bloodstream infections (BSI) were observed to be most frequently associated on the medical wards.

Also, BSI was mostly observed on the paediatric medical wards, whereas skin and soft tissue infection (SSTI) was most frequently observed on the surgical wards. Nosocomial central nervous system infections and nosocomial urinary tract infections were more commonly observed on the medical facilities followed by surgical wards. Nosocomial urinary tract infection was least observed on the gynaecology wards. Candida albicans, Enterobacter and Escherichia coli isolates were most commonly observed in urinary tract infections.

Of the susceptibility test results, Amoxicillin (AML), Amoxicillin/Clavulanic acid (AMC), Cefaclor (CEC), Cefuroxime (CXM), Ampicillin (AMP) and Trimethoprine/Sulphamethazole (SXT) were observed of being the most frequently resistant antibiotics in this study. Resistance of these antibiotics were commonly observed for the following nosocomial pathogens: Acinetobacter, Klebsiella,

Staphylococcus, Enterobacter, Escherichia coli, Serratia sp. and Citrobacter koserii organisms. In the literature has been published that the highest resistance rate was against Ciprofloxacin and Imipenem in Iran [52]. A total of eight multi-resistant strains of gram-negative bacteria with ESBL-production were detected in five E. coli and three K. pneumoniae strains at the Albert Schweitzer hospital in Gabon. However, four were resistant to the whole spectrum of antibiotics available [27]. Van der Zee A et al

(2014) reported that the resistance to Carbapenem antibiotics is emerging worldwide among Enterobacteriaceae and they developed a PCR technique for identification of carbapenemase genes: blaOXA-48, blaVIM, blaIMP, blaNDM and blaKPC in cultures of broth rectal swabs [53]. Lin et al (2014) reported that Tigecycline non-susceptible K. pneumoniae bacteraemia may suggest a critical problem that caused high mortality of patients at a medical centre in Taiwan over a 3-year period. It was divulged that resistance to commonly used antibiotics was observed in up to $80 \%$ of the isolates in Malawi [54].

However, Pseudomonas aeruginosa, Acinetobacter and Klebsiella were observed to be most frequently susceptible to Imipenem, Amikacin, Ciprofloxacin, Gentamicin and Cefepime. Quinupristin

(QD) was observed to be most commonly susceptible for methicillinresistant coagulase negative Staphylococcus (MRCNS) organisms and Clindamycin, Rifamficin, Tigercycline, Vancomycin and Linezolid were 
also commonly observed to be effective for methicillin-resistant coagulase negative

Staphylococcus (MRCNS) isolates. Sulphamethazole was frequently observed to be susceptible for Citrobacter koserii, Stenotrophomonas maltophilia and Alcaligenes sp. In contrast Sulphamethazole was least susceptible for Burkholderia (P) cepacia isolates. Piroth L et al, 2014 reported that $\mathrm{E}$. coli were susceptible to third-generation Cephalosporins and amoxicillin-clavulanate in $89.5 \%$ and $62.5 \%$ of cases, respectively. No single antibiotic allowed antimicrobial coverage of more than $60 \%$ in 190 cirrhotic patients in France [55]. Natoli S et al, 2009 reported that bacteraemia caused by Coagulase negative Staphylococci remained susceptible to Linezolid, Daptomycin and Tigecycline in Italy [56].

In this study inpatients were more likely of being associated with nosocomial infections due to inconsistency in sanitizing work areas, lack of proper ventilation on some wards, irregularity in changing long term use of invasive devices such as urinary and central venous catheters, long term stays in hospitals and previous hospitalization exposure. Patients acquired nosocomial infections either endogenously or from external environment (exogenously). Endogenous nosocomial infections were because of opportunistic pathogens residing in or on external surfaces of patients and brought on by conditions present at or as a direct outcome of events on the wards. In contrast, exogenous hospital acquired infections were the result of pathogens being transmitted by patients as they are shed from numerous thresholds of exit while the patients were hospitalized. Zilberberg MD et al, 2014 reported several risk factors that predispose to recurrent Clostridium difficile infection including use of certain antimicrobial such as Fluoroquinolones and IV Vancomycin after completion of $\mathrm{C}$. difficile treatment, community-onset healthcare associated infection, $\geq 2$ hospitalizations within prior 60 days, age and gastric acid suppression [11,57].

Among the reported risk factors for colonization by extensively drugresistant $P$. aeruginosa in immunocompromised patients, the unnecessary use of antibiotics, particularly ciprofloxacin was reported; and when using medical devices, it was suggested that a high standard of infection control measures must be achieved [28, 58,76]. Yamakawa K et al, 2011 reported that Healthcare-associated methicillin resistant Staphylococcus aureus infection in ICU patients causes high mortality, which is associated to risk factors such as intubation, treatment with antibiotics, open wound, and steroid administration, all occurring within 24 hours of ICU admission [12-13, 59]. Vasudevan A et al, 2014 agreed with previous authors that the global increase of resistant gram-negative bacilli infections in ICU are due to various risk factors and the most important one is the widespread use of empiric broad spectrum antibiotics $[1,60]$.

In this study the infection control measures practiced at the health care facilities as recorded from patient's dockets during weekly surveillance included: isolation of MRSA patients. These individuals were separated from others to avoid non-affected inpatients becoming contaminated. It was observed wearing gloves and face masks by the barrier nurses when attending to MRSA patients. Other infection control measures that were observed at the health care facilities included strict observance of contact precautions, ventilatory support, and use of antibiotics, analgesia, fluid resuscitation and frequent cleaning of infected wounds with Lysol. All were part of the management of the sick documented by health care professionals. In addition, it was advised that indwelling catheters be changed frequently to prevent persistence and reoccurrence of infections. Nosocomial Infections pose serious health problems or challenges to patient well-being. Therefore, CDC provides world-wide guidance in close watch, outbreak surveys, laboratory research and prevention of nosocomial infections. CDC uses awareness acquired through these campaigns to spot infections and develop and implement new plans to prevent and reduce nosocomial infections. Public health action by CDC and other healthcare partners demonstrated great improvements in clinical practice, medical methods and the continuing growth of infection control guidance and prevention accomplishments.

Hospitals should comply with all sanitization protocols comprising uniforms, fumigating equipments, washing and other pre-emptive procedures. Proper hand washing along with usage of alcohol rubs by all health care staff prior to and after each patient contact is one of the most effective ways of fighting hospital-acquired infections. These measures have been observed by other researchers [62-68]. Furthermore, some health personnel have challenged the idea that the stethoscope, may essentially be a path for transmission of nosocomial infections. In a research of a hundred and fifty (150) health care staffs, fifty (50) paramedics, fifty (50) nurses, and fifty (50) doctors, Staphylococcus sp., mostly coagulase negative were cultured from $89 \%$ of the participant's stethoscopes with the average amount of colony forming units increased, when the stethoscopes were not sanitized [5, 69]. In general, $48 \%$ of health care workers cleaned their stethoscopes each day or week, $37 \%$ each month, $7 \%$ annually and $7 \%$ had never cleaned them. Cleaning the stethoscope caused an immediate decline in the bacterial count by $94 \%$ with alcohol swabs, 90\% with a non-ionic detergent, and $75 \%$ with antibacterial soap [69]. With regards to ventilators, which may be an aid to prevent the risk of hospital-acquired pneumonia infections by controlling hospital internal air quality must be a fulfilled requirement at the most suitable areas. To an extent nosocomial infection can be lessened to strengthen this point as reported by [70].

Cautious use of antibiotics is crucial. Despite sanitation guidelines, patients can become susceptible of acquiring hospital-acquired infections and they are often given alternative antibiotics in controlling infections that may amplify the range for the appearance of resistant strains. In addition, sterilization is further than sanitizing; it destroys all pathogens on medical devices and surfaces via contact with chemicals, ionizing radiation, dry heat or steam under pressure. Safety measures must be implemented to avoid spread of pathogens by regular paths in health care facilities.

Practicing of hand washing is the solitary way of minimizing the hazards of spreading skin pathogens between patients or from one spot to another on the same patient. Frequent hand washing as often between contacts with patients and after contact with blood, body fluids, secretions, excretions, and equipment or items infected by these pathogens is a vital constituent of infection management and isolation preventative measures. The transmission of hospital-acquired infections, among immunocompromised patients is linked with health care staff's hand infectivity in nearly $40 \%$ of cases and it is a difficult predicament in the modern health care facilities. The most appropriate application for staffs to conquer this challenge is performing proper hand-hygiene actions.

Therefore, the World Health Organization (WHO) initiated in 2005, the Global Patient Safety Challenge. The objective of hand sanitation is to eradicate the transitory flora with proper act of hand washing, with various types of soap; customary and antibacterial and alcohol-based gels. The major challenges observed in the performance of hand sanitation are associated with the inadequacy of sinks, lengthy time and act of hand washing procedures. A simple way in resolving this issue can be using alcohol-based hand rubs, since, it is a quicker process in contrast to accurate hand washing [71].

\section{Conclusion}

Consistency in performing good hygiene practices is vital for reducing the high nosocomial rate found at the research sites. Prediction of these infections is very important as a part of clinical surveillance programs to take preventive measures in advance. ASPR showed that only $8.3 \%$ $(5 / 60)$ of the isolates were antibiotic-susceptible strains. 


\section{Acknowledgements}

This study was self-sponsored. I would like to thank Alexander Elliott for the financial support making this project a reality and God for His sustaining grace throughout the successful completion of this research.

\section{Competing Interests}

All authors declare that they have no competing interests.

\section{Author Contributions}

CE made contributions to the study design, data acquisition, analysis and interpretation of data. She also drafted the manuscript. AJV took part in analysis, writing up and revision of the manuscript. All authors read and approved the final manuscript.

\section{References}

1. Yoshioka N, Deguchi M, Hagiya H, Yoshida H, Yamamoto N, Hashimoto $\mathrm{S}$ et al. (2018) Comprehensive (ABC) score to a diagnosis of Methicillin-resistant Staphylococcus aureus infection: a derivation and validation study. BMC Infect Dis.18: 19.

2. Zhou F, Li H, Gu L, Liu M, Xue CX, Cao B, et al. (2018) National Influenza A (H1N1) pdm09 Clinical Investigation Group of China. Risk factors for nosocomial infection among hospitalized severe influenza A (H1N1) pdm09 patients. Respir Med. 134: 86-91.

3. Seifu WD, Gebissa AD. (2018) Prevalence and antibiotic susceptibility of Uropathogens from cases of urinary tract infections (UTI) in Shashemene referral hospital, Ethiopia. BMC Infect Dis. 18: 30.

4. Goto T, Yoshida K, Tsugawa Y, Filbin MR, Camargo CA Jr, Hasegawa K. (2016) Mortality trends in U.S. adults with septic shock, 2005-2011: a serial cross-sectional analysis of nationally representative data. BMC Infect Dis. 16: 294.

5. Iordanou S, Middleton N, Papathanassoglou E, Raftopoulos V. (2017) Surveillance of device associated infections and mortality in a major intensive care unit in the Republic of Cyprus. BMC Infect Dis.17: 607.

6. Anago E, Ayi-Fanou L, Akpovi CD, Hounkpe WB, Agassounon-Djikpo TM, Bankole HS, Sanni A. (2015) Antibiotic resistance and genotype of beta-lactamase producing Escherichia coli in nosocomial infections in Cotonou, Benin. Ann Clin Microbiol Antimicrob.14:5.

7. Zhang H, Yang Q, Liao K, Ni Y, Yu Y, Hu B, et al. (2017) Update of incidence and antimicrobial susceptibility trends of Escherichia coli and Klebsiella pneumoniae isolates from Chinese intraabdominal infection patients. BMC Infect Dis.17: 776.

8. Cheddie P, Dziva F, Akpaka PE. (2017) Detection of a CTX$M$ group 2 beta-lactamase gene in a Klebsiella pneumoniae isolate from a tertiary care hospital, Trinidad and Tobago. Ann Clin Microbiol Antimicrob.16: 33.

9. Orrett, F A. (2002) "Nosocomial Infections in an Intensive Care Unit in a Private Hospital." West Indian Medical Journal.21-24.

10. Frank DN, Wilson SS, St Amand AL, Pace NR. (2009) Culture-independent microbiological analysis of Foley urinary catheter biofilms. PLoS One 4: e7811.

11. Ulrich RJ, Santhosh K, Mogle JA, Young VB, Rao K. (2017) Is Clostridium difficile infection a risk factor for subsequent bloodstream infection? Anaerobe.48: 27-33.
12. Johnson NB, Hayes LD, Brown K, Hoo EC, Ethier KA. (2014) Centres for Disease Control and Prevention (CDC). CDC National Health Report: leading causes of morbidity and mortality and associated behavioural risk and protective factors-United States, 2005-2013. MMWR Suppl.63: 3-27.

13. Doyle JS, Buising KL, Thursky KA, Worth LJ, Richards MJ. (2011) Epidemiology of infections acquired in intensive care units. Epidemiology of infections acquired in intensive care units. Semin Respir Crit Care Med.32: 115-138.

14. Ho TS, Wang SM, Wu YH, Shen CF, Lin YJ, Lin CH, et al. (2010) Long-term characteristics of healthcare-associated infections in a neonatal intensive care unit. J Microbiol Immunol Infect.43: 407-415.

15. Sato M, Kubota N, Katsuyama Y, Suzuki Y, Miyairi Y, Minami K, et al. (2017) Case report of a 6-yearold girl with Mycoplasma hominis ventriculoperitoneal shunt infection. J Neurosurg Pediatr.19: 620-624.

16. Raymond J, Aujard Y. (2000) Nosocomial infections in pediatric patients: a European, multicentre prospective study. European Study Group. Infect Control Hosp Epidemiol.21: 260-263.

17. Klevens RM, Edwards JR, Richards CL Jr, Horan TC, Gaynes RP, Pollock DA, et al. Estimating health care-associated infections and deaths in U.S. hospitals, 2002. Public Health Rep.122: 160-166.

18. Wang PH, Wang HC. (2016) Risk factors to predict drugresistant pathogens in haemodialysis-associated pneumonia. BMC Infect Dis. 16: 377.

19. American Thoracic Society. (2005) Infectious Diseases Society of America. Guidelines for the management of adults with hospital-acquired, ventilator-associated, and healthcareassociated pneumonia. Am J Respir Crit Care Med.171: 388416.

20. Doyle JS, Buising KL, Thursky KA, Worth LJ, Richards MJ. (2011) Epidemiology of infections acquired in intensive care units. Epidemiology of infections acquired in intensive care units. Semin Respir Crit Care Med.32: 115-138.

21. Whitson WJ, Ball PA, Lollis SS, Balkman JD, Bauer DF. (2014) Postoperative Mycoplasma hominis infections after neurosurgical intervention. J Neurosurg Pediatr.14: 212-218.

22. Stevens DL, Bisno AL, Chambers HF, Dellinger EP, Goldstein EJ, Gorbach SL, et al. (2014) Infectious Diseases Society of America. Practice guidelines for the diagnosis and management of skin and soft tissue infections: 2014 update by the Infectious Diseases Society of America. Clin Infect Dis.59:10-52.

23. Deshpande LM, Fritsche TR, Moet GJ, Biedenbach DJ, Jones RN. (2007) Antimicrobial resistance and molecular epidemiology of vancomycin-resistant enterococci from North America and Europe: a report from the SENTRY antimicrobial surveillance program. Diagn Microbiol Infect Dis.58:163-170.

24. Nadimpalli M, Stewart JR, Pierce E, Pisanic N, Love DC, Hall D, et al. (2016) Livestock-Associated, Antibiotic-Resistant Staphylococcus aureus Nasal Carriage and Recent Skin and Soft Tissue Infection among Industrial Hog Operation Workers. PLoS One.11: e0165713.

25. Rubin MA, Samore MH, Harris AD. (2018) The Importance of Contact Precautions for Endemic Methicillin-Resistant Staphylococcus aureus and Vancomycin-Resistant Enterococci. JAMA.Feb 12.

26. Ahoyo TA, Bankolé HS, Adéoti FM, Gbohoun AA, Assavèdo S, Amoussou-Guénou M, et al. (2014) Prevalence of nosocomial infections and anti-infective therapy in Benin: 
results of the first nationwide survey in 2012. Antimicrob Resist Infect Control.3:17.

27. Scherbaum M, Kösters K, Mürbeth RE, Ngoa UA, Kremsner PG, Lell B, et al. (2014) Abraham Alabi. Incidence, pathogens and resistance patterns of nosocomial infections at a rural hospital in Gabon. BMC Infect Dis.14: 124.

28. Rosenthal VD, Maki DG, Graves N. (2008) The international nosocomial infection control consortium (INICC): goals and objectives, description of surveillance methods, and operational activities. Am J Infect Control.36: 1-12.

29. Ducel $\mathrm{G}$ et al. Guide pratique pour la lutte contre l'infection hospitalière. WHO/BAC/79.1.

30. Benenson AS. Control of communicable diseases manual, 16th edition. Washington, American Public Health Association, 1995.

31. World Health Organization. Prevention of hospital-acquired infections: A practical guide. $2^{\text {nd }}$ edition. 2002. Retrieved from the WHO/CSR Web site on 20/2/2018.

32. Wayne PA. Performance Standards for Antimicrobial Susceptibility Testing. Clinical and Laboratory Standards Institute, 2014.

33. Lauren Hund, Edward J. (2015) Bedrick, Marcello Pagano. Choosing a Cluster Sampling Design for Lot Quality Assurance Sampling Surveys. PLoS One.10: e0129564.

34. Peronnet E, Nguyen K, Cerrato E, Guhadasan R, Venet F, Textoris J, et al. (2016) Evaluation of mRNA Biomarkers to Identify Risk of Hospital Acquired Infections in Children Admitted to Paediatric Intensive Care Unit. PLoS One.11: e0152388.

35. Strausbaugh LJ. (2001) Emerging health care-associated infections in the geriatric population. Emerg Infect Dis.MarApr; 7: 268-271.

36. Garibaldi RA. (1999) Residential care and the elderly: the burden of infection. J Hosp Infect.43 Suppl: S9-18.

37. Engelhart ST, Hanses-Derendorf L, Exner M, Kramer MH. (2005) Prospective surveillance for healthcare-associated infections in German nursing home residents. J Hosp Infect.60: 4650.

38. Tsan L, Davis C, Langberg R, Hojlo C, Pierce J, Miller M, et al. (2008) Prevalence of nursing home associated infections in the Department of Veterans Affairs nursing home care units. Am J Infect Control.36: 173-179.

39. Marzahn D, Pfister W, Kwetkat A. (2017) Influence of nosocomial infections on activities of daily living in acute geriatric inpatients. Z Gerontol Geriatr.

40. Girard R, Gaujard S, Pergay V, Pornon P, Martin-Gaujard G, Bourguignon L. (2017) UTIC Group. Risk factors for urinary tract infections in geriatric hospitals. J Hosp Infect.97: 74-78.

41. Bourdel-Marchasson I, Kraus F, Pinganaud G, TexierMaugein J, Rainfray M, Emeriau JP. (2001) Annual incidence and risk factors for nosocomial bacterial infections in an acute care geriatric unit Rev Med Interne.22: 1056-1063.

42. Girard R, Gaujard S, Pergay V, Pornon P, Martin-Gaujard G, Bourguignon L; UTIC Group. Risk factors for urinary tract infections in geriatric hospitals. J Hosp Infect.97: 74-78.

43. Stenlund M, Sjödahl R, Pia Yngman-Uhlin RN. Incidence hospital acquired. Int J Qual Health Care. 2017 Apr 1; 29: 290294.

44. GlobalSurg Collaborative. Surgical site infection after gastrointestinal surgery in high-income, middle-income, and low-income countries: a prospective, international, multicentre cohort study. Lancet Infect Dis.Feb 13.

45. Mazaki T, Mado K, Masuda H, Shiono M. (2013) Antibiotic prophylaxis for the prevention of surgical site infection after tension-free hernia repair: a Bayesian and frequentist metaanalysis. J Am Coll Surg.217: 788-801.1-4.

46. Ali S, Birhane M, Bekele S, Kibru G, Teshager L, Yilma Y, et al. (2018) Healthcare associated infection and its risk factors among patients admitted to a tertiary hospital in Ethiopia: longitudinal study. Antimicrob Resist Infect Control.7: 2.

47. Ruppen C, Notter J, Strahm C, Sonderegger B, Sendi P. (2018) Osteoarticular and skin and soft-tissue infections caused by Streptococcus agalactiae in elderly patients are frequently associated with bacteraemia. Diagn Microbiol Infect Dis.90: 55-57.

48. Reichmann DE, Greenberg JA. (2009) Reducing Surgical Site Infections: A Review. Rev Obstet Gynecol.2: 212-221.

49. Erol S, Zenciroğlu A, Dilli D, Okumuş N, Aydin M, Göl N, et al. (2014) Evaluation of nosocomial blood stream infections caused by Pseudomonas species in new-borns. Clin Lab.60: 61520.

50. Jefferies JM, Cooper T, Yam T, Clarke SC. (2012) Pseudomonas aeruginosa outbreaks in the neonatal intensive care unit-a systematic review of risk factors and environmental sources. J Med Microbiol.61:1052-1061.

51. Baş AY, Demirel N, Zenciroglu A, Göl N, Tanir G. (2010) Nosocomial blood stream infections in a neonatal intensive care unit in Ankara, Turkey. Turk J Pediatr.52: 464-70.

52. Rajabi M, Abdar ME, Rafiei H, Aflatoonia MR, Abdar ZE. Nosocomial Infections and

53. Epidemiology of Antibiotic Resistance in Teaching Hospitals in South East of Iran. Rajabi M, et al. Glob J Health Sci. 2015.

54. Roorda L, Bosman G, Hermans M, Witt R, Stuart JC, Ossewaarde JM. (2014) Multi-centre evaluation of real-time multiplex PCR for detection of carbapenemase genes OXA-48, VIM, IMP, NDM and KPC. BMC Infect Dis.14: 27.

55. Yi-Tsung L, Yu-Ping W, Fu-Der W, Chang-Phone F. (2015) Community-onset Klebsiella pneumoniae pneumonia in Taiwan: clinical features of the disease and associated microbiological characteristics of isolates from pneumonia and nasopharynx. Front Microbiol. 6: 122.

56. Piroth L, Pechinot A, Di Martino V, Hansmann Y, Putot A, Patry I, et al. (2014) Evolving epidemiology and antimicrobial resistance in spontaneous bacterial peritonitis: a two-year observational study. BMC Infect Dis.14: 287.

57. Natoli S, Fontana C, Favaro M, Bergamini A, Testore GP, Minelli S, et al. (2009) Characterization of coagulase-negative staphylococcal isolates from blood with reduced susceptibility to glycopeptides and therapeutic options. BMC Infect Dis.9: 83.

58. Zilberberg MD, Reske K, Olsen M, Yan Y, Dubberke ER. (2014) Risk factors for recurrent Clostridium difficile infection (CDI) hospitalization among hospitalized patients with an initial CDI episode: a retrospective cohort study. BMC Infect Dis. 14: 306.

59. Willmann M, Klimek AM, Vogel W, Liese J, Marschal M, Autenrieth IB, et al. (2014) Clinical and treatment-related risk factors for nosocomial colonization with extensively drugresistant Pseudomonas aeruginosa in a haematological patient 
population: a matched case control study. BMC Infect Dis.14: 650 .

60. Yamakawa K, Tasaki O, Fukuyama M, Kitayama J, Matsuda H, Nakamori Y, et al. (2011) Assessment of risk factors related to healthcare-associated methicillin-resistant Staphylococcus aureus infection at patient admission to an intensive care unit in Japan.BMC Infect Dis.

61. Vasudevan A, Mukhopadhyay A, Li J, Yuen EGY, Tambyah PA. (2014) A prediction tool for nosocomial multi-drug resistant gram-negative bacilli infections in critically ill patients - prospective observational study. BMC Infect Dis.14: 615.

62. Prevention of hospital-acquired infections: A practical guide. 2nd edition. 2002. WHO/CSR Web site. http://apps.who.int/medicinedocs/documents/s16355e/s16355 e.pdf (Viewed 25/2/2018).

63. Geffers C, Gastmeier P. (2011) Nosocomial infections and multidrug-resistant organisms in Germany: Epidemiological data from KISS (the hospital infection surveillance system) Dtsch Arztebl Int.108: 87-93.

64. Dogru A, Sargin F, Celik M, Sagiroglu AE, Goksel MM, Sayhan H. (2010) The rate of device associated nosocomial infections in a medical surgical intensive care unit of a training and research hospital in Turkey: one-year outcomes. Jpn J Infect Dis.63: 95-98.

65. Sax H, Allegranzi B, Chraiti MN, Boyce J, Larson E, Pittet D. (2009) The world health organization hand hygiene observation method. Am J Infect Control.37: 827-834.

66. Garner JS, Jarvis WR, Emori TG, Horan TC, Hughes JM. (1991) CDC definitions for nosocomial infections 1988. Z Arztl Fortbild (Jena).85: 818-827.

67. Horan TC, Andrus M, Dudeck MA. (2008) CDC/NHSN surveillance definition of health care-associated infection and criteria for specific types of infections in the acute care setting. Am J Infect Control.36: 309-332.

68. Rosenthal VD, Guzman S, Safdar N. (2005) Reduction in nosocomial infection with improved hand hygiene in intensive care units of a tertiary care hospital in Argentina. Am J Infect Control.33: 392-397.

69. Salmon S, Pittet D, Sax H, McLaws ML. (2015) The 'My five moments for hand hygiene' concept for the overcrowded setting in resource-limited healthcare systems. J Hosp Infect.91: 95-99.

70. Jones JS, Hoerle D, Riekse R. (1995) Stethoscopes: a potential vector of infection? Ann Emerg Med.26: 296-9.
71. Leung M, Chan AH. (2006) Control and management of hospital indoor air quality. Med Sci Monit.12: SR17-23.

72. Hugonnet S, Perneger TV, Pittet D. (2002) Alcohol-based hand rub improves compliance with hand hygiene in intensive care units. Arch Intern Med.13; 162: 1037-1043.

73. Berenholtz SM, Pronovost PJ, Lipsett PA, Hobson D, Earsing K, Farey JE et al. (2004) Eliminating catheter-related bloodstream infections in the intensive care unit. Crit Care Med. 32:2014-2020.

74. Pronovost P, Needham D, Berenholtz S, Sinopoli D, Chu H, Cosgroe S, et al. (2006) An intervention to decrease catheterrelated bloodstream infections in the ICU. N Eng J Med.355: 2725- 2732.

75. Rosenthal VD, Jarvis WR, Jamulitrat S, Silva CP, Ramacandran B, Duenas L, et al. (2012) Socioeconomic impact on device-associated infections in pediatric intensive care units of 16 limited-resource countries: international nosocomial infection control consortium findings. Pediatr Crit Care Med.13: 399-406.

76. Higuera F, Rangel-Frausto MS, Rosenthal VD, Soto JM, Castanon J, Franco G, et al. (2007) Attributable cost and length of stay for patients with central venous catheter-associated bloodstream infection in Mexico City intensive care units: a prospective, matched analysis. Infect Control Hosp Epidemiol.28: 31-35.

77. National Committee for Clinical Laboratory Standards, author. Tentative Guidelines, M26TNCCLS. Villanova, PA: 1993. Methods for determining bactericidal activity of antimicrobial agents.

78. Yuan Y, Zhang Y, Fu S, Crippen TL, Visi DK, Benbow ME, et al. (2017) Genome sequence of a Providencia stuartii strain isolated from Lucilia sericata salivary glands. Genome Announc.5: e00250-17.

79. Hughes D, Andersson DI. (2017) Environmental and genetic modulation of the phenotypic expression of antibiotic resistance. FEMS Microbiol Rev.41: 374-391.

80. Yu-Tin C, Hwei-Ling P, Wei-Chung S, Fang-Rong H, ChuianFu K, Yu-Ming T, et al. (2012) Whole genome and identification of Morganella morganii KT pathogenicityrelated genes. BMC Genomics.13: S4.

81. Sousa SA, Ramos CG, Leitão JH. (2011) Burkholderia cepacia Complex Emerging Multihost Pathogens Equipped with a Wide Range of Virulence Factors and Determinanta. Int $\mathbf{J}$ Microbiol.2011: 607575.

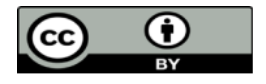

This work is licensed under Creative Commons Attribution 4.0 License

To Submit Your Article Click Here: Submit Manuscript

DOI: $10.31579 /$ brcr.2020/001
Ready to submit your research? Choose Auctores and benefit from:

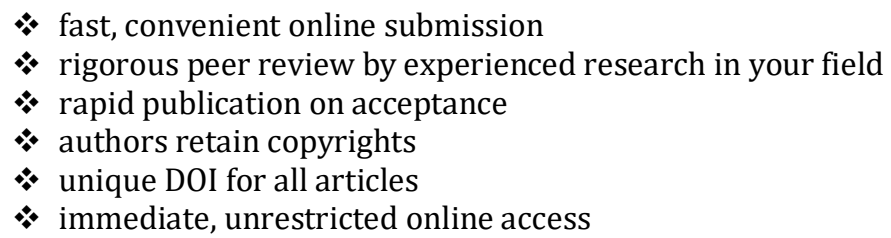

At Auctores, research is always in progress.

Learn more www.auctoresonline.org/journals/biomedical-research-andclinical-reviews- 